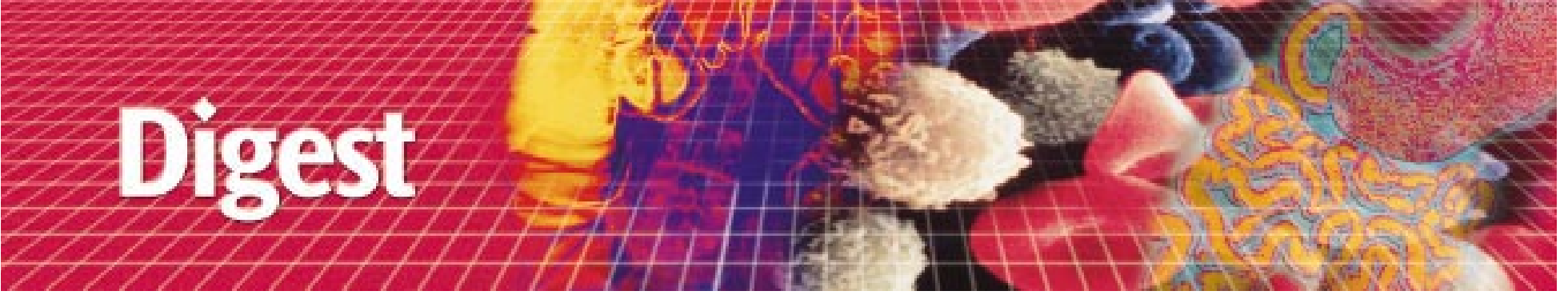

Ian Forgacs, Associate Editor

\section{IBD: A PREGNANT PAUSE}

There are a few misconceptions about inflammatory bowel disease (IBD) and pregnancy. Two reports in this month's Gut help to clarify our understanding. Most women with IBD will have a normal outcome of their pregnancy. Our editorial suggests that commonly used drugs are "safe". Yet the conclusions of a Danish study "strongly link IBD with adverse birth outcome". Readers should look closely at the confidence intervals around the risk factors and then draw their own conclusions.

See pages 159 and 243

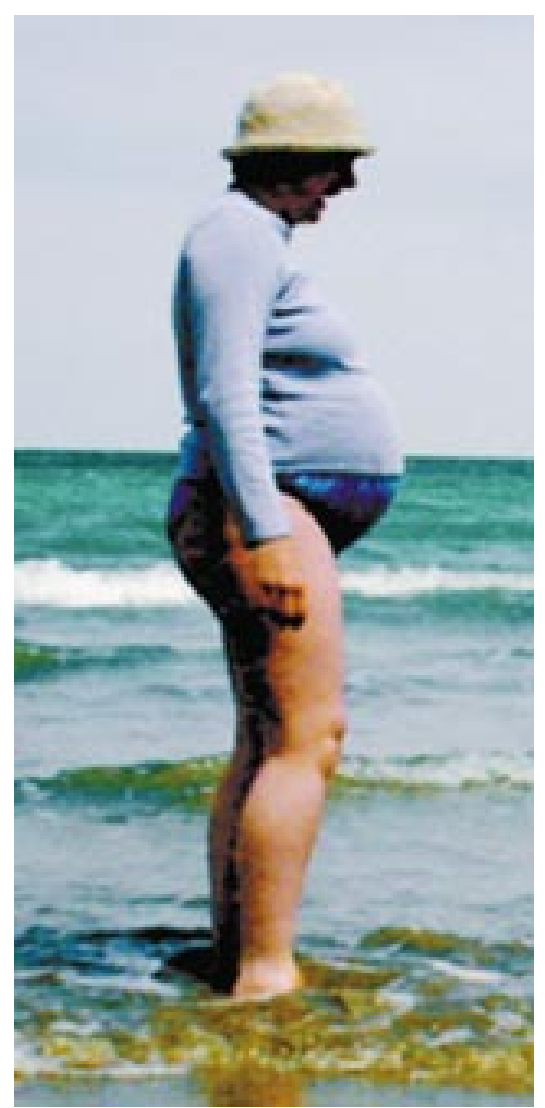

\section{H PYLORI AND PEPTIC ULCER: A LEISURE ACTIVITY}

Some readers might be surprised to find a paper in a 21 st century issue of Gut on risk factors for peptic ulceration. Yet

www.gutjnl.com maybe it is timely to re-evaluate the time honoured factors that predispose to ulcer disease in the H pylori era. There are few surprises and it's certainly no shock to find smoking included on the list. But it seems "moderate leisure time physical activity" powerfully protects against peptic ulcer disease in those with $H$ pylori. Read the paper to find out how what constitutes moderate activity in Denmark. The possibility of a confounding factor certainly exercises the mind.

See page 186

\section{MASS SCREENING OR MASS HYSTERIA}

The observed prevalence of coeliac disease has been increasing steadily in the past 20 years-maybe " 1 in 200 " or even "approximately $1 \%$ " of the population have the condition. Coeliac disease is undeniably common-no argument about that, but Gut debates whether screening is justified. Fasano suggests coeliac disease meets WHO guidelines for mass screening of the population; Kumar thinks otherwise. Opponents in a debate should marshal the most cogent arguments in favour of their respective views. Debates inevitably polarise opinion so if you wish to contribute, do feel free to visit our website at www.gutjnl.com.

See pages 168 and 170

\section{BUDESONIDE FINDS A NICHE}

Budesonide seems to have been embraced with varying degrees of enthusiasm by clinicians who treat inflammatory bowel disease. It may, however, be really useful in collagenous colitis. Unlike too many drugs that show promise in uncontrolled series but fail the ordeal by randomised trial, budesonide has proved highly effective in a placebo controlled, double blind study of patients with collagenous colitis. The reduction in stool weight and frequency was remarkable, the histological improvement was also most impressive. Budesonide supporters will also rejoice that their favourite drug may have a beneficial effect on enhancing sugar uptake after intestinal resection. The study was in rats but there may be implications for humans.

See pages 248 and 252

\section{COMBINATION, CLEARANCE, AND CURE}

Without prophylaxis, hepatitis $C$ virus is detectable in most patients transplanted because of chronic HCV liver disease. Combination therapy with interferon-ribavirin may eradicate the virus, normalise liver function, and improve liver histology, yet the long term benefit is not established. Bizollon and colleagues studied 54 patients with recurrent HCV infection post-transplant who had been treated with combination therapy. Complete response (loss of graft HCV-RNA) was sustained in $93 \%$ of those patients tested a mean of three years after treatment had finished. Such patients may be considered to be cured of the infection.

See page 283

\section{TRYPSIN: A REGULATOR OF INNATE INTESTINAL IMMUNITY.}

The mechanisms that protect and defend the intestine against bacterial assault have been shown to be of considerable complexity and sophistication. It is rather surprising and no less pleasing to find an enzyme one associates inextricably with the pancreas to be a player in regulating intestinal immunity. Alpha defensins, which are expressed mainly in neutrophils and Paneth cells, constitute $70 \%$ of released bactericidal activity in some species such as the mouse. In man, Paneth cells express two defensins (HD5 and 6). HD5 is stored as a propeptide which turns out to be activated by trypsin cleavage. This month's Science@lert describes in more detail how an old friend seems to have a rather intriguing "new" role.

See page 166 